\title{
LXII. Crystallographic notes
}

\section{Fletcher M.A.}

To cite this article: L. Fletcher M.A. (1882) LXII. Crystallographic notes, Philosophical Magazine Series 5, 13:84, 474-480, DOI: 10.1080/14786448208627218

To link to this article: http://dx.doi.org/10.1080/14786448208627218

\section{曲 Published online: 28 Apr 2009.}

Submit your article to this journal 준

LII Article views: 2

Q View related articles ¿ 
These give illustrations of the results accomplished; but of course many other experiments have been made. I have not yet been able to decide whether the definition of the concave grating fully comes up to that of a flat grating; but it evidently does so very nearly.

Baltimore, May 25, 1882.

LXIT. Crystallographic Notes. By L. FLeTCHer, M.A., of the Mineral Department, British Museum*.

[Plate X.]

IX. Skuttenudite.

THE first mention of this mineral was made in 1827 by 1 Breithauptt, to whom it had been submitted by his brother-in-law on returning from a voyage in Norway. Though none of the specimens presented crystal-faces, Breithaupt found that there were distinct cleavages parallel to the sides of a cube, for which reason he assigned to the species the name of Tesseral-Kies. Cleavages, more or less interrupted and indistinct, were found to exist parallel to planes truncating the edges and quoins of this cube, indicating that the crystallisation was that characteristic of the cubic system. Breithaupt went a step further, and, from traces of separation which manifested themselves in the direction of the octahedral planes, hazarded the conjecture, since (curious to say) verified, that the crystals would prove to present the octahedron as the predominant form. The specimens were of a bright metallic lustre and of a tin-white colour. The specific gravities of five different fragments were determined to be $6.659,6 \cdot 681,6.718,6.748,6.848$, and thus had a considerable range. As, however, after breaking up the fragments, it was seen that the first three included particles of quartz and actinolite, Breithaupt regarded the true specific gravity as lying between 6.748 and $6 \cdot 848$. The hardness was greater than that of cobaltite or Cobaltkies; and as blowpipe examination had indicated that cobalt and arsenic were the chief constituents, Breithaupt suggested the secondary title of Hartkobaltkies. The associated minerals were cobaltine, copper pyrites, glassy actinolite, serpentine, quartz, and sometimes also cobalt-bloom.

* Communicated by the Author, having been read before the Crystallological Suciety, May 30, 1881.

† "Ueber eine neue Kies-Species von Skutterud," Pogg. Ann. rol. ix. p. $115(1827)$. 
Ten years later Scheerer* met with a mineral which examination led him to regard as identical with that previously described by Breithaupt. A quantitative nnalysis, afterwards confirmed by Wöhlert, gave the following result:-

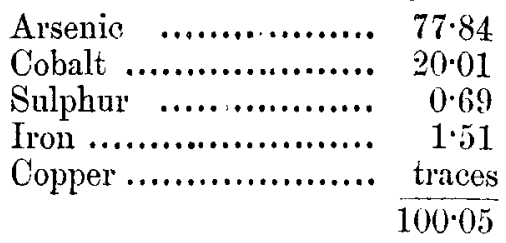

a composition expressed by the formula CoAs.

The description given by Scheerer agrees very closely with that of Breithaupt; but not only did he find the mineral in its massive condition, but also as isolated crystals. These had the form of octahedra, modified by the cube dodecahedron and an icositetrahedron: the inclination of the faces of the latter form to the adjacent face of the octahedron, as measured, was $19^{\circ} 27^{\prime}$, proving clearly that the icositetrahedron was (2 11 ), for which the calculated angle is $19^{\circ} 28^{\prime}$; the faces of this particular icositetrahedron truncate the edges of the dodecahedron. He further observes that this form (211) was present on every crystal he had examined. Scheerer notices also the interesting fact that the crystals are often found implanted upon crystals of cobaltite, and states that he had not been able to trace any regularity in the relative position of the crystals of the two species. As the term Tesseralkies might be taken to imply that the mineral crystallises in the form of cubes, he suggests Arsenikkobaltkies as a more appropriate name.

In Haidinger's 'Handbuch der bestimmenden Mineralogie' (1845) the mineral appears under still another title-Skutterudite, suggestive of the locality where the crystals are found.

In Brook and Miller's edition of 'An Elementary Introduction to Mineralogy by the late William Phillips,' published in 1852 , the forms presented by Skutterudite are given as (100), $(110),(111)$, and (221), and the angles according with this description are calculated. It will be observed that the form (2 11 ) described by Scheerer is not mentioned, but that a new form, (221), a triakisociahedron, is substituted. In 1862 rom Rath $\ddagger$ apparently not having referred to the

* "Ueber zwei nor wegische Kobalterze ron den Skntteruder Gruben," Pogg. Ann. vol. xlii. p. 553 (1837).

† Pogg. Ann. vol. liii. p. 591.

f "Nene Flächen am Tesseralkies," Pogg. Amm. vol. cxv. p. 480. 
original paper of Scheerer, and assuming that the information contained in the last-mentioned 'Mineralogy' incorporated all the results of observation up to that date, determined anew the form (211), and also added a triakisoctahedron (332), a tetrakishexahedron (310), and a hexakisoctahedron (643).

The prominent forms, according to both Scheerer and vom Rath, are the octahedron (111), the dodecahedron (110), and the icositetrahedron (211); while, according to the latter mineralogist, the additional forms (332), $(310),(643)$ are only subordinately developed. The disposition of the faces of this hexakisoctahedron (643) will be more easily imagined, if it be remarked that they cut off the edges of intersection of the octahedron with the tetrakishexahedron (310). With regard to the faces of the triakisoctahedron, vom Rath observes that they are small and unsuited for measurement, but that in one case he had been able to measure the angle made with the adjacent face of the octahedron as lying between $9 \frac{1}{2}^{\circ}$ and $10 \frac{1}{2}^{\circ}$; whence he concludes that the form is not $(221)$, as given by Miller, which requires an angle of $15^{\circ} 48^{\prime}$, but (332), for which the calculated angle is $10^{\circ} 2^{\prime}$.

As vom Rath considered it unlikely that the subordinate triakisoctahedron present on some crystals should be (221) and on others (332), he suggests that the symbol given by Miller is a mistaken one, and appears to think that the form (221) may have been determined by inspection and not by measurement. It seems, however, much more probable that the information given by Miller is intended to be merely a statement of the results of Scheerer, and not of a later examination by Miller himself, who could not have failed to remark the existence of the icositetrahedron (211), which is so characteristic that it was present on every crystal examined by Scheerer, and in fact can be distinguished on every specimen in the collection of the British Museum. It seems fairly evident, then, that Miller's (221) was a simple error introduced in the copying of Scheerer's results, and that the angles were afterwards calculated without this error in the translation of the symbol from the German notation being: remarked. If this hypothesis be correct, (221) must be expelled from the list of observed forms.

The next mention of Skutterudite is to be found in an extremely interesting and important paper by Schrauf and Dana on the thermoelectric properties of mineral-varieties*, where they remark that, although the mineral undoultedly crystallises in the cubic system, and has never been observed to

" "Veber die thermoelektrische Eigenschaften von Mineral-Varietäten," Sitz.-Ber. Ak. Wien, vol, lxix. p. 153 (1874). 
present any hemihedral development, they nevertheless find some specimens to be thermoelectrically positive and others negative.

The bearing of this remark will be more obvious when it is recalled to mind that the investigation of Schrauf and Dana was undertaken with a view to test the accuracy of the induction made by Gustav Rose from his experiments on iron pyrites and cobaltine* - that the opposition in the thermoelectric behaviour of different specimens of the same mineral is due to the diplohedral hemisymmetry,-and of his inference that the faces observed on the positive and negative crystals belong only apparently to the same, really to complementary, semiforms. The observation of Schrauf and Dana, that the same thermoelectrical peculiarities are shown by a holohedral mineral of the same crystalline system to which iron pyrites and cobaltine belong, will clearly not strengthen the position taken up by Rose.

It is therefore of some interest to find that the specimens in this Collection prove that not only is Skutterudite undoubtedly hemihedral, but that it presents the particular variety of bemisymmetry which is so characteristic of both iron pyrites and cobaltine.

Brezinat has pointed out that the opposition of thermoelectric properties shown by various specimens of iron pyrites and cobaltine cannot be satisfactorily explained by diplohedral symmetry, and holds that haplohedral hemisymmetry must be at the same time present-that, in other words, the crystals must be tetartohedral in structure; and, assuming that the differences in the specimens are only differences of molecular grouping, Brezina regards iron pyrites and the allied species as being really tetartohedral-a view which as yet there is little, if any, crystallographic evidence to support. It seems, however, more probable that Schrauf and Dana are right in attributing this difference, not to crystalline hemisymmetry, but to slight differences of composition or of density; and the force of their argument will not be very much weakened by the reference of the symmetry of the crystalline forms of Skutterudite to a hemihedral type.

It may be useful to remark that attention was first directed to the hemihedry by the discovery of a crystal of Skutterudite among the specimens of that typically hemihedral mineral

* "Ueber den Zusammenhang zwischen hemiëdrischer Krystallform und thermo-elektrischen Verhaiten beim Eisenkies und Kobaltglanz," Pogg. Ann. vol, cxlii. p. 1 (1871).

† “Ueber die Symmetrie der Pyritgruppe," Tschermak's Min, Mittheil. p. $23(1872)$. 
cobaltine, to which it was very similar in habit, though different in colour. Closer examination, however, made known the fact that the hemihedrally-developed faces belonged, not to the pentagonal dodecahedron $\pi(210)$, so common in both cobaltine and iron pyrites, but to the much rarer form $\pi(310)$. In this Collection there are fourteen more or less perfect crystals, presenting respectively the following development:-

No. 1 shows only the octahedron $o(111)$ modified by faces of the icositetrahedron $n(211)$, as shown in fig. 1 (Pl. X.).

Nos.2,3,4 show also the faces of the dodecahedron $d(110)$, and are represented in fig. 2-each of these four crystals being implanted upon a crystal of cobaltite in the way described by Scheerer and vom Rath. On Nos. 5 and 6 the same forms recur; but some of the quoins are truncated by small planes of the cube $a(100)$, probably due to cleavage.

As in all the above forms the poles of the faces lie in dodecahedral planes, the abeyance of symmetry of the latter would bave no effect in reducing the number of faces; in other words, so long as only these forms are present it is impossible to distinguish crystallographically whether the structure is characterized by holohedral symmetry or by diplohedral hemisymmetry.

Nos. 7 to 11 present faces of the tetrakishexahedron $f(310)$; and it now becomes possible to determine whether the internal structure as shown by the external form is to be regarded as holohedral or hemihedral. As a matter of fact, in each of these crystals the faces of only one semiform $\pi(310)$ are found to be present, the number varying with the more or less fractured state of the crystal. This combination is represented in fig. 3, from which, for the sake of simplicity, the small faces of the cube have been omitted. No. 7 presents all the twelve faces required by perfect hemisymmetry; No. 8 shows eight faces, No. 9 shows five, while on Nos. 10 and 11 only two are present; but on none of these crystals can any faces of the complementary semiform $\pi(130)$ be distinguished.

Crystal No. 12 is five eighths of an inch ( $=1.6$ centim.) long, and projects from a matrix of quartz and mica. It is particularly interesting as showing the hemihedral development, not only of the above tetrakishexahedron, but also of an hexakisoctahedron having its planes in the edge-zones of the dodecahedron. The angle made by the faces of this new form with the adjacent faces of the form (211) was measured by help of the telescopic images to be $10^{\circ} 48^{\prime}$ in one case and $11^{\circ} 2^{\prime}$ in another ; there is no doubt, then, that the faces belong to the semiform $\pi$ (312), for which the corresponding calculated angle is $10^{\circ} 54^{\prime}$. Fig. 4 represents the actual development of the various faces on that part of the crystal 
Phil .Mag. S. 5.Vol.13.Pl.X

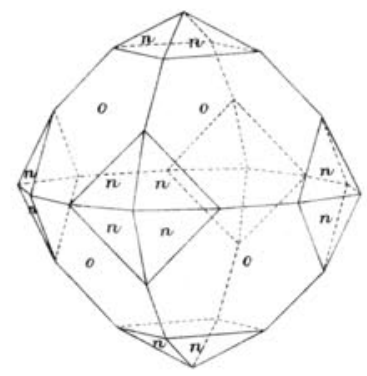

F ig: 1 .

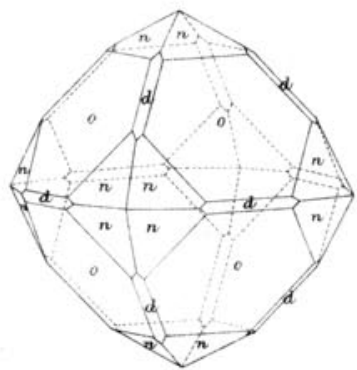

F ig: 2 .

$$
\begin{array}{ll}
o & 111) \\
d & (110) \\
a & (100) \\
n & (211) \\
f & \pi(310) \\
s & \pi(312)
\end{array}
$$

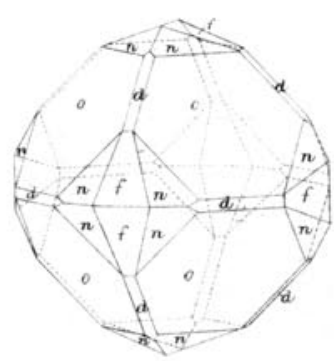

Fig: 3 .

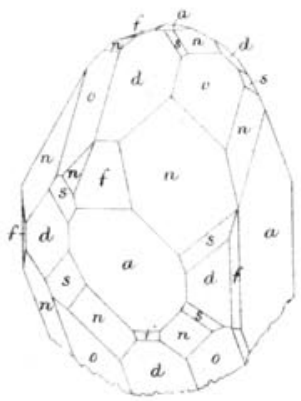

Fig: 4. 
which is not concealed by the matrix : no fewer than ten faces of this semiform $\pi(312)$ can be seen, while not a single face of the complementary semiform $\pi(321)$ is to be found.

Gustar Rose has remarked that, in cases of diplohedral hemisymmetry, the poles of all the faces present belong in general to one set of systematic triangles: this crystal of Skutterudite forms another of the rare exceptions to this rule, which would require the association of $\pi(321)$ instead of $\pi(312)$ with the semiform $\pi(310)$.

This semiform $\pi(312)$ is again to be observed on crystal No. 13, though it is there represented by only a single face. The angle with the adjacent face of the form (211) was in this case measured to be $10^{\circ} 52 \frac{1}{2}$, a result according well with the calculated angle $10^{\circ} 54^{\prime}$ : on the same crystal eight faces of the semiform $\pi(310)$ are developed.

The last crystal, No. 14, differs from the rest in showing three faces, which, if the crystal were simple, would undoubtedly be attributed to the complementary semiform $\pi(130)$; the crystal, however, still presents a hemihedral habit, since the three faces of this complementary semiform $\pi(130)$ only appear at quoins where the faces of $\pi(310)$ are missing. It is very possible indeed that the crystal may be twinned about the normal to a dodecahedron-face, as is at times the case in iron pyrites-which theory would likewise account for the presence of reentrant angles, otherwise to be attributed to parallel growth.

The subordinate forms (332), (643), described by vom Rath, do not seem to be present on any of the above crystals, and must be very rare. In some cases indeed the edges of intersection of the octahedron with the form $\pi(310)$ are " rounded off" by very small faces not susceptible of measurement; while the edges of the octahedron itself are bevelled by narrow planes, which, if crystal-faces at all, can only be approximately determined. On one crystal the angles made with the adjacent octahedral faces by the four tautozonal rudimentary planes of the triakisoctahedron were measured by the method of maximum illumination as $18 \frac{1}{2}^{\circ}, 17 \frac{1}{4}^{\circ}, 13^{\circ}$, $16 \frac{1}{2}^{\circ}$ respectively - thus suggesting the form (221), which requires an angle of $15^{\circ} 48^{\prime}$, and, as we have seen, was given by Miller, no doubt in mistake, as an observed form. On another crystal a series of images could be obtained from each of two faces of the triakisoctahedron; and the limiting-values thus determined for the angle corresponding to the one just mentioned were, in one case $13 \frac{1}{2}^{\circ}-16 \frac{1}{4}^{\circ}$, and in the other $153^{\circ}-16 \frac{1}{2}^{\circ}$; on a third crystal two similar angles were measured at $15 \frac{1}{4}^{\circ}$ and $19^{\circ}$, thus again indicating the form (2 21$)$, 
The range of specific gravity of the crystallised mineral is a little wider than that of the massive as determined by Breithaupt: one crystal presenting no external sign of impurity was found to have a specific gravity of 6.48 ; a second had a specific gravity of $6 \cdot 37$, but, like the less dense specimens of Breithaupt, evidently contained foreign matter. Two other fairly large crystals had specific gravities of 6.56 and 6.72 respectively; while a small apparently very pure crystal, with smooth lustrous faces, gave a result as high as $6 \cdot 86$.

LXIII. Action of Free Molecules on Radiant Heat, and its Conversion thereby into Sound. By JoHN Trndalu, F.R.S.

[Concluded from p. 462.]

Contents.

Page

Sect. 5. New Experiments. Hypothesis of Interual Films .. 480

a. The Experimental Tube ................ 480

$b$. The Plates of Rock-salt . ............... 486

6. Conservation of Molecular Action . . . . . . . . . . 491

7. Thermal Continuity of Liquids and Vapours ..... 495

8. Rhythmic Absorption of Radiant Heat by Gases and

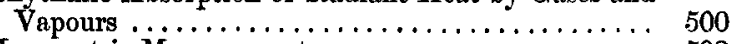

9. Manometric Measurements ................. 508

10. Application of Results to Meteorology ......... 514

\$5. New Eaperiments. Hypothesis of Internal Films.

a. The Experimental Tube.

I

WISH now to come to closer quarters with the argument urged by Magnus and repeated by MM. Lecher and Pernter, namely that my results were due to "vapourhesion "- that is to say, to liquid films condensed on my experimental tube and on my plates of rock-salt. The two investigators last named express unqualified surprise that I should have neglected the simple precaution of experimenting with blackened tubes. But this precaution was by no means neglected by me. I have repeatedly fortified myself by experiments of this character, with the result recorded in Table I. But I went further. A smooth coating of lampblack, however powerful as an absorber, might be competent to reflect a eertain portion of the incident heat. Hence my desire to get entirely rid of reflection, by avoiding all contact with the interior surface of the experimental tube.

In fig. 3 an apparatus is sketched, by means of which this has been accomplished. $\mathrm{T} \mathrm{T}^{\prime}$ is a stout tube of brass, 36 inches long and 6 inches internal diameter. Projecting from 